\title{
Surface Molecular Imprinted Electrochemical Sensor for Lysozyme Based on Metal Coordinate Technology
}

\author{
${\text { Zhonghe } \mathrm{CHEN}^{1,2, \mathrm{a}} \text {, Mingbo BAO }}^{2, \mathrm{~b}}$, Yuchuan $\mathrm{LI}^{1, \mathrm{c}}, \mathrm{Zhi} \mathrm{CHEN}^{2, \mathrm{~d}}$ \\ ${ }^{1}$ Shandong Provincial Key Laboratory of Fluorine Chemistry and Chemical Materials \\ ${ }^{2}$ School of Chemistry and Chemical Engineering, University of Jinan \\ Jinan, China

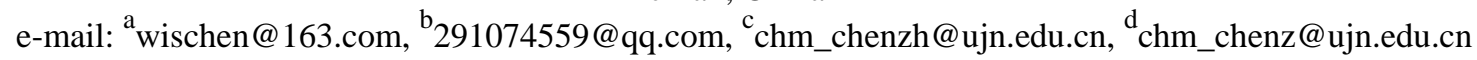

\begin{abstract}
A novel and efficient molecular imprinted polymers (MIPs) electrochemical sensor based on metal coordination technology was prepared by electro-polymerization, which was applied as a molecular recognition element to modify glassy carbon electrode (GCE) to construct an electrochemical sensor (MIPs/IDA/GR/GCE) for sensitive detection of lysozyme (Lyz). The prepared MIPs/IDA/GR/GCE showed a fast rebinding dynamics, which was successfully applied to Lyz detection with a wide linear range from $1.0 \times 10^{-11}$ to $1.0 \times 10^{-5} \mathrm{~g} / \mathrm{L}(\mathrm{R}=0.998)$ and a detection limit of $2.3 \times 10^{-12} \mathrm{~g} / \mathrm{L}$. Moreover, the fabricated sensor possessed a good selectivity and sensitivity, providing a promising tool for immunoassays and clinical applications.
\end{abstract}

Keywords-electrochemical sensor; metal coordinate technology; lysozyme; electro-polymerization

\section{INTRODUCTION}

Molecular imprinting technology (MIT) offers considerable potential for biomolecular-based recognition due to its advantages of chemical and thermal stability, reusability, fabrication, and high recognition capacity to the template molecule in a variety of sensor applications. ${ }^{1-4}$ Molecular imprinting electrochemical sensor (MIECS), which combined the MIT and electrochemical sensor, inhibited high selectivity and sensitivity by using molecular imprinting polymers (MIPs) as specific recognition element for template molecule. Up to now, MIECSs have been successfully used to imprint small biomolecules. ${ }^{5,6}$ But it still remains a challenge for the imprinting of protein because of the decrease of sensitivity, due to the complexity of protein structure such as large molecular size and flexible conformation. Among the techniques used for protein imprinting, surface imprinting is currently the most popular and general method to solve the problem of diffusion limitation caused by large size of protein.

As we know, metal ion coordination plays important role in biological recognition systems, since many pharmaceutics demonstrate biological activities when present in the form of metal complexes ${ }^{77]}$. Metal chelation is reversible valence bond force under certain condition, which makes metal ions and biological molecules can be combined or fractured under mild conditions, they could be used to promoted biological molecular imprinting ${ }^{[8,9]}$. Metal ion-mediated MIP has been demonstrated to have high selectivity and has been exploited in several types of selective recognition systems. ${ }^{10-12}$ Metal ion-mediated imprinting is of great interest to introduce enhanced functionality into recognition sites of MIPs, depending on the metal, its oxidation state, ligand characteristics, and the strength of interaction can vary enormously. ${ }^{13}$ Metal ions are known to bind functional groups through the sharing of electrons from the atoms of templates to the unfilled orbitals of the outer coordination sphere of the metal. ${ }^{14,15}$ So, we combine the MIT and metal coordinate technology to fabricate complex imprinted polymer (CIP), in which metal ion mediated imprinting may impart more stability in water and protic solvents owing to inherent covalent coordinations, in comparison to the traditional approach. Bereli et al. prepared a Lyz imprinted supermacroporous cryogel by using N-methacryloyl-(L)histidinemethylester (MAH) as the metal-coordination monomer complexes with $\mathrm{Cu}^{2+}-\mathrm{Lyz}^{16}$

Graphene (GR), a two-dimensional (2D) crystal of sp2hybridized carbon atoms arranged in six-membered rings, has become a popular material in the development of electrochemical sensors and biosensors due to its unique physicochemical properties, such as fast electron transfer, large surface area, high stability, excellent adsorptive and biocompatibility. ${ }^{17-20}$ Recently, GR has been used for the preparation of MIECSs to improve sensitivity. ${ }^{21-24}$

There is strong interaction among $\mathrm{Cu}^{2+}$, disulfide bond and N-three peptides in Lyz. ${ }^{25}$ Herein, a novel and efficient MIPs electrochemical sensor based on metal coordination technology was prepared by electro-polymerization. In which, graphene was used as sensitization material, and modified imino diethyl acetic acid (IDA) which could interact with Lyz to immobilize the template molecule Lyz on modified electrode to fabricate molecular imprinted electrochemical sensor (MIPs/IDA/GR/GCE). The property and the performance of the proposed MIPs/IDA/GR/GCE were investigated by electrochemical impedance spectroscopy (EIS) and differential pulse voltammetry (DPV). The fabricated sensor indicated excellent selectivity and anti-interference capability for detection of Lyz. Compared with the traditional detector, the MIPs/IDA/GR/GCE was simple, with low limit of detection, high sensitivity and stability. 


\section{EXPERIMENTAL}

\section{A. Reagents and apparatus}

Lysozyme (Lyz) and pyrrole were purchased from Sinopharm Chemical Reagent Co. Ltd. (Shanghai, China). Bovine hemoglobin (BHb), bovine serum albumin (BSA) and Cytochrome $\mathrm{C}$ (Cyt-c) were provided from the Shuangxuan Microorganism Substrate Plant of Beijing. Graphite was friendly provided by Qingdao Fujin graphite Co. Ltd. (Qingdao, China). Graphene was synthesized according to the methods mentioned in Wang's reported work. ${ }^{20}$ Imino diethyl acetic acid (IDA) was provided from the Shuangxuan Microorganism Substrate Plant of Beijing. All other reagents used were of analytical grade and used as received without further purification. All solutions were prepared with doubly distilled water throughout the experiment. The electrochemical measurements were all performed with a CHI 6V60C electrochemical workstation (Shanghai, China). The classical three-electrode system consisted of an IDA modified graphene based Lyz molecular imprinted glassy carbon electrode (GCE) as the working electrode, a potassium chloride $(\mathrm{KCl})$-saturated calomel electrode (SCE) as the reference electrode, and a Pt wire electrode as the auxiliary electrode; Shanghai Kudos SK5200H ultrasonic cleaner. All electrochemical experiments were performed in $\mathrm{PBS}(\mathrm{pH}=7.0)$ containing $0.1 \mathrm{mM}\left[\mathrm{Fe}(\mathrm{CN})_{6}\right]^{3-/ 4-}$.

\section{B. Fabrication of IDA/GR/GCE}

Before modification, the glassy carbon electrode (GCE) was polished carefully with 0.3 and $0.5 \mathrm{~m} \mathrm{Al}_{2} \mathrm{O}_{3}$ slurry successively. And then the GCE was ultrasonically cleaned in doubly distilled water and ethanol for a few seconds, followed by drying before use. The IDA/GR/GCE was made by dropping $5 \mu \mathrm{L}$ of GR-IDA mixed solution (10.0 mg GR and $13.3 \mathrm{mg}$ IDA were dissolved in $10 \mathrm{~mL}$ doubly distilled water) on the GCE surface and then heated under an IR lamp to remove the solvent.

\section{Fabrication of MIPS/IDA/GR/GCE}

The as-prepared IDA/GR/GCE was immersed into deoxygenated PBS containing Lyz $(1.0 \mathrm{~g} / \mathrm{L})$ and pyrrole $(\mathrm{pH}=7.0)$ and $0.05 \mathrm{mM} \mathrm{CuSO}_{4}$, incubated $20 \mathrm{~min}$ to form IDA- $\mathrm{Cu}^{2+}$-Lyz complex completely, and then followed by cyclic voltammetry $(\mathrm{CV})$ at the scanning rate of $100 \mathrm{mV} / \mathrm{s}$ for 5 cycles under a potential from $-0.2 \mathrm{~V}$ to $1.2 \mathrm{~V}$ to get the polymers modified IDA/GR/GCE (Lyz@MIPs/IDA/ GCE). After the electro-polymerization, the polymers modified electrode was incubated into $1 \mathrm{M} \mathrm{H}_{2} \mathrm{SO}_{4}$ for $2 \mathrm{~h}$, respectively, to extract the template protein Lyz to obtain the imprinted IDA/GR/GCE (MIPs/IDA/GR/GCE). The procedure for the preparation of the MIPs/IDA/GR/GCE is depicted in Scheme 1. As a contrast, MIPs/GR/GCE was prepared exactly as above but without the modification of IDA.

\section{Electrochemical property measurements}

$\left[\mathrm{Fe}(\mathrm{CN})_{6}\right]^{3-/ 4-}$, which can diffuse into and out of imprinted caves of imprinted polymer film to reach modified electrode and produce an electrochemical signal because of the oxidation or reduction on the electrode, was used as an electrochemical active probe to study the response of Lyz on the prepared imprinted sensor. The MIPs/IDA/GR/GCE was immersed into Lyz solution with different concentrations, and incubated for $20 \mathrm{~min}$ to ensure protein molecule rebound by MIPs/IDA/GR/GCE. DPV method was conducted for electrochemical determination of Lyz in PBS $(\mathrm{pH}=7.0)$ containing $0.1 \mathrm{~m} \mathrm{M}\left[\mathrm{Fe}(\mathrm{CN})_{6}\right]^{3-/ 4-}$ from 0.0 to $0.4 \mathrm{~V}$ at a scan rate of $100 \mathrm{mV} / \mathrm{s}$. All electrochemical experiments were carried out at room temperature. BSA, BHb and Cyt-C were chosen as control proteins to evaluate the selectivity of the MIPs/IL/GR/GCE.

\section{Results AND Discussion}

\section{A. Characteristics of MIPs/IDA/GR/GCE}

The surface morphologies of the Lyz@MIPs/IDA/ GCE (with template molecular) and MIPs/IDA/GR/GCE (without template molecular) were evaluated by scanning electron microscopy (SEM). There was great difference in the morphologies between Lyz@MIPs/IDA/GR/GCE and MIPs/IDA/GR/GCE. As shown in Fig. 1A, a layer of film was coated on the surface of IDA/GR/GCE with the electropolymerization of the MIPs. Fig. 1B showed the morphologies of MIPs/IDA/GR/GCE which removed the template molecular. Compare with Fig. 1A, it showed a rather rough and dense surface and some pores were left in the MIPs layer to provide high-speed pathways for the template molecules transfer from solution to the sensor.

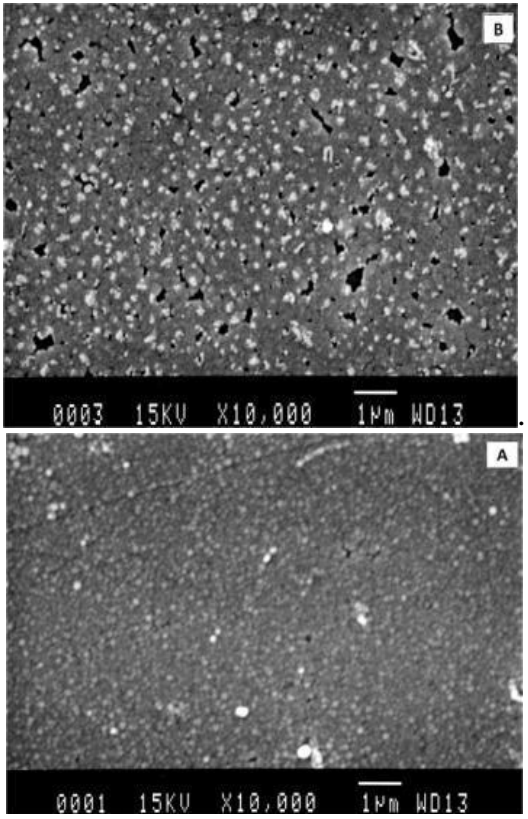

Figure 1. SEM images of Lyz@MIPs/IDA/GR/GCE (with template molecular) (A) and MIPs/IDA/GR/GCE (without template molecular) (B). 


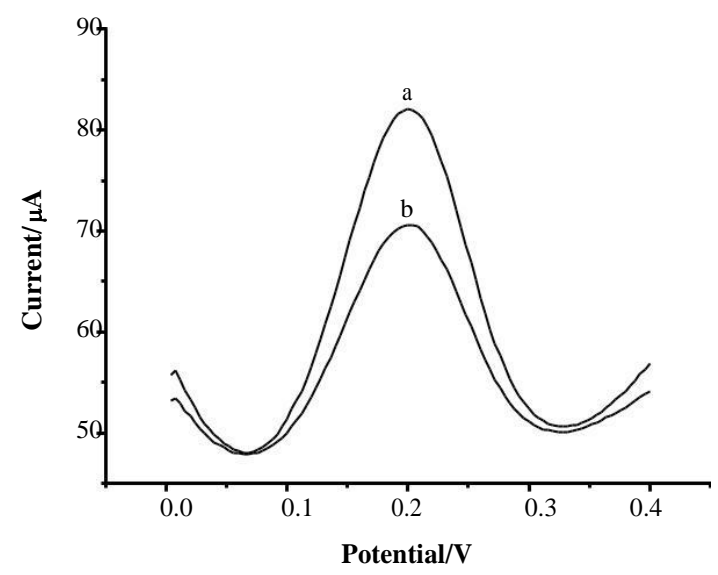

Figure 2. Typical DPVs of (a) MIPs/IDA/GR/GCE and (b) MIPs/GR/GCE in pH 7.0 PBS containing $0.1 \mathrm{mM}\left[\mathrm{Fe}(\mathrm{CN})_{6}\right]^{3-1 / 4}$. Scan rate $100 \mathrm{mV} / \mathrm{s}$.

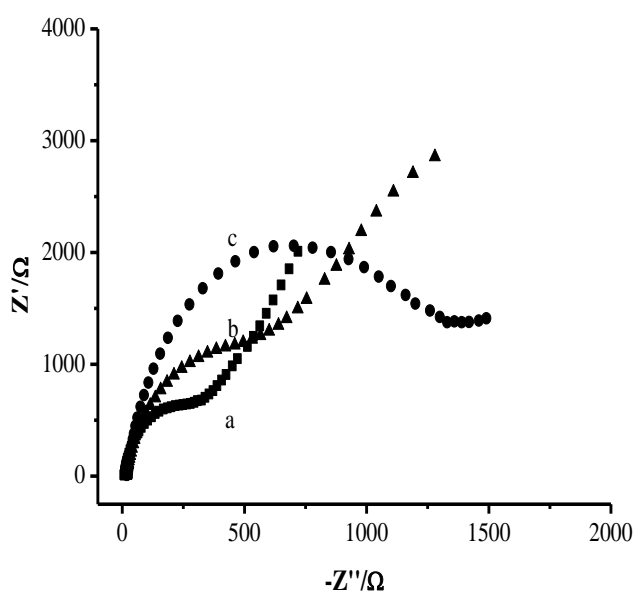

Figure 3. Electrochemical impedance spectroscopy (EIS) of (a) MIPs/IDA/GR/GCE, (b) MIPs/IDA/GR/GCE rebounded with Lyz and (c) Lyz@MIPs/IDA/GR/GCE in pH 7.0 PBS containing $0.1 \mathrm{mM}$ $\left[\mathrm{Fe}(\mathrm{CN})_{6}\right]^{3-/ 4-}$ at frequencies from 0.01 to $10^{5} \mathrm{~Hz}$.

In order to investigate the performance of fabricated molecular imprinted electrochemical sensor, we measured the DPVs of different molecular imprinted electrodes (MIPs/IDA/GR/GCE,MIPs/GR/GCE) in $\mathrm{pH} \quad 7.0$ PBS containing $0.1 \mathrm{mM}\left[\mathrm{Fe}(\mathrm{CN})_{6}\right]^{3-14}$. As shown in Fig. 2, compared with MIPs/GR/GCE, we could find bigger peak current on MIPs/IDA/GR/GCE, which was attributed to the introduction of $\mathrm{Cu}^{2+}$. There was metal coordination interaction between $\mathrm{Cu}^{2+}$ and $\mathrm{Lyz}$, which could immobilize more template molecules on IDA/GR/GCE than GR/GCE, leading to more imprinted caves after elution resulting more channels for the penetration of $\left[\mathrm{Fe}(\mathrm{CN})_{6}\right]^{3-/ 4-}$ through the polymer film to reach the IDA/GR/GCE for further oxidation.

As an effective method for probing the features of a surface-modified electrode, electro-chemical impedance spectrum (EIS) could be applied to characterize the stepwise construction process of the sensor. Fig. 3 represented the EIS of MIPs/IDA/GR/GCE (curve a), MIPs/IDA/GR/GCE rebounded with Lyz (curve b) and Lyz@MIPs/IDA/ GCE (curve c). When the template protein Lyz was removed out of the imprinted polymer film, the electron transfer resistance was much smaller than that of Lyz@MIPs/IDA/GR/GCE, was attributed to the elution of template protein BSA resulting in the increment of electron transfer rate and conductivity. As protein Lyz is not electroactive, it can hinder the electron transfer rate resulting in bigger electron transfer resistance. Imprinted caves were formed after eluting the template protein Lyz, which could be permeating channel for electrochemical probe diffusing through the polymer film to modified electrode surface. The changes of electron transfer rate in Fig. 3 illustrated that template protein Lyz had been eluted successfully and formed imprinted caves with special recognition.

\section{B. The performance of MIPS/IDA/GR/GCE}

In order to illustrate the efficiency of prepared MIPs/IDA/GR/GCE, the adsorption dynamics experiment was carried out by recording the current responses of MIPs/IDA/GR/GCE and MIPs/GR/GCE incubated in Lyz solution $\left(1.0 \times 10^{-4} \mathrm{~g} / \mathrm{L}\right)$ for different time. The MIPs/IDA/GR/GCE possessed a higher and faster current responses (curve a, $32.8 \mu \mathrm{A}$ and $60 \mathrm{~min}$ ) compared with that of MIPs/GRR/GCE (curve b, $21.0 \mu \mathrm{A}$ and $120 \mathrm{~min}$ ) at the same condition, indicating the excellent sensitivity and speedy balance response were ascribed to the introduction of $\mathrm{Cu}^{2+}$, which increased and accelerated the immobilization of Lyz to the fabricated molecular imprinted electrochemical sensor. This proposed strategy could be a promising technique for rapid detection of protein in spot specimens in clinical application.

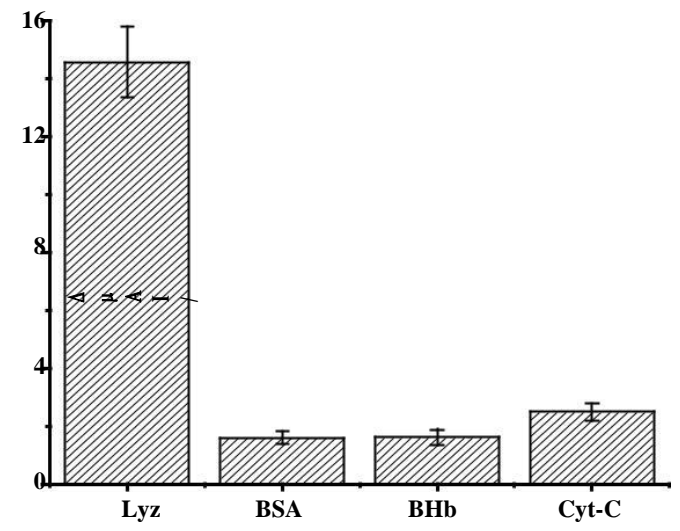

Figure 4. The selectivity of MIPs/IDA/GR/GCE for Lyz, BSA, BHb and Cyt-C. The concentration of each compound is $1.0 \times 10^{-7} \mathrm{~g} / \mathrm{L}$.

In order to estimate the selectivity of the MIPs/IDA/GR/GCE for Lyz analysis, BSA, BHb and Cyt-C were used as control proteins. The concentration of each molecule is $1.0 \times 10^{-7} \mathrm{~g} / \mathrm{L}$. The corresponding changes of current response (A) on MIPs/IDA/GR/GCE determined by 
DPV method. As shown in Fig. 4, the $\Delta \mathrm{I}$ on MIPs/IDA/GR/GCE toward Lyz was the highest, which 9.4, 9.4 and 5.8 times of that toward BSA, BHb, Cyt-C, respectively. These data demonstrated the high selectivity of fabricated MIPs/IDA/GR/GCE for the electrochemical detection of the template protein Lyz.

Moreover, the prepared MIPs/IDA/GR/GCE was used for the determination of Lyz with different concentrations to investigate the sensitivity of the molecular imprinted electrochemical sensor. As shown in Fig 6, the $\Delta \mathrm{I}$ on MIPs/IDA/GR/GCE decreased with the increasing Lyz concentrations from $1.0 \times 10^{-11}$ to $1.0 \times 10^{-5} \mathrm{~g} / \mathrm{L}$. A good linear regression equation can be expressed as $\Delta \mathrm{I}(\mu \mathrm{A})=$ $38.87+3.42 \log$ CLyz $(\mathrm{g} / \mathrm{L})(\mathrm{R}=0.998)$ with the limit of detection is $2.3 \times 10^{-12} \mathrm{~g} / \mathrm{L}$. These results demonstrated obviously that the fabricated MIPs/IDA/GR/GCE possessed excellent sensitivity for Lyz determination.

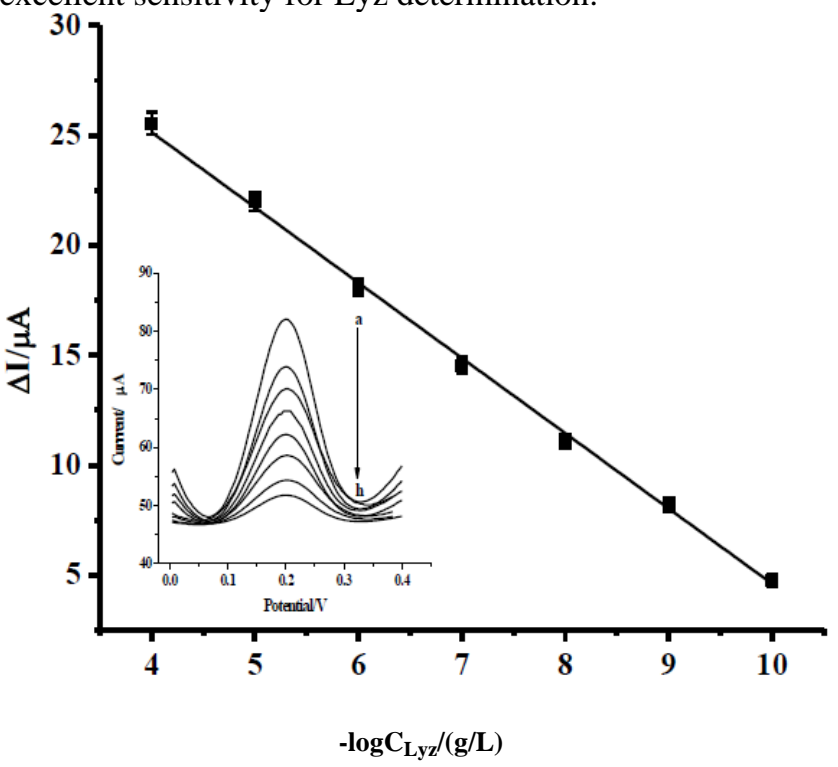

Figure 5. Calibration curve of the prepared MIPs/IDA/GR/GCE for various concentrations of Lyz in $\mathrm{pH} 7.0 \mathrm{PBS}$ containing $0.1 \mathrm{mM}$ $\left[\mathrm{Fe}(\mathrm{CN})_{6}\right]^{3-1 / 4}$. (Insert: corresponding DPV curves a $\rightarrow$ h: blank, $1.0 \times$

$$
\begin{gathered}
10^{-11}, 1.0 \times 10^{-10}, 1.0 \times 10^{-9}, 1.0 \times 10^{-8}, 1.0 \times 10^{-7}, 1.0 \times 10^{-6}, 1.0 \times \\
10^{-5} \mathrm{~g} / \mathrm{L}, \text { respectively.). }
\end{gathered}
$$

The repeatability of MIPs/IDA/GR/GCE was also investigated. The relative standard deviation (RSD) was $3.0 \%$ from the responses of three successive measurements in $\mathrm{pH} 7.0$ PBS containing $0.1 \mathrm{mM}\left[\mathrm{Fe}(\mathrm{CN})_{6}\right]^{3-/ 4-}$, which indicated that the proposed MIPs/IDA/GR/GCE possessed a good repeatability. The molecular imprinted electrochemical sensor also exhibited satisfactory stability. In fact, as much as $95 \%$ of the initial peak current was preserved after storage of the molecular imprinted electrochemical sensor at $4{ }^{\circ} \mathrm{C}$ for 15 days.

\section{CONCLUSION}

A novel and efficient MIPs electrochemical sensor based on metal coordination technology was prepared by electropolymerization. The prepared MIPs/IDA/GR/GCE showed excellent sensitivity, high selectivity, excellent stability and fast balance response for the electrochemical determination of template protein Lyz. Therefore, the proposed strategy could be a promising technique for quantitative detection of protein in clinical application

\section{ACKNOWLEDGMENT}

We greatly appreciate the financial support from the National Natural Science Foundation of China (21405086, 21475071, 21275082, 81102411 and 21203228), the National Key Basic Research Development Program of China (973 special preliminary study plan, Grant no. 2012CB722705), the Taishan Scholar Program of Shandong Province and the Natural Science Foundation of Qingdao (13-1-4-128-jch and 13-1-4-202-jch).

\section{REFERENCES}

[1] L. Chen, S. Xu and J. Li, Chem Soc Rev, 2011, 40, 2922-2942.

[2] D. Yu, Y. Zeng, Y. Qi, T. Zhou and G. Shi, Biosens Bioelectron, 2012, 38, 270-277.

[3] D. Cai, L. Ren, H. Zhao, C. Xu, L. Zhang, Y. Yu, H. Wang, Y. Lan, M. Roberts, J. Chuang, M. Naughton, Z. Ren and T. Chiles, Nat Nanotechnol, 2010, 5, 597-601.

[4] V. Suryanarayanan, C. Wu and K. Ho, Electroanalysis, 2010, 16, 1795-1811.

[5] Z. Wang, F. Li, J. Xia, L. Xia, F. Zhang, S. Bi, G. Shi, Y. Xia, J. Liu, L. Xia, Biosens Bioelectron, 2014, 61, 391-396.

[6] B. Lu, J. Xia, Z. Wang, F. Zhang, M. Yang and Y. Li, RSC Adv., 2015, 5, 82930-82935.

[7] B. Prasad and I. Pandey, Sensors and Actuators B-Chemical, 2013, $186,407-416$

[8] K. Dhal, F and Arnold, Macromolecules, 1992, 25, 7051-7059.

[9] L. Zhu, R. Zhang and Z. Zhu, Chemistry Online, 2010, 4, 326-331.

[10] L. Wu and Y. Li, Anal Chim Acta, 2003, 482, 175-181.

[11] S. Sreenath, A. Sergey, V. Elena, B. Chen, K. Karim and A. Turner. Biosens Bioelectron, 2001, 16, 631-637.

[12] N. Brunkan and M. Gagné. J. Am. Chem. Soc., 2000, 122, 6217 6225.

[13] H. EL-Sharif, H. Yapati, S. Kalluru and S. Reddy. Acta Biomaterialia, 2015, 28, 121-127.

[14] C. Alexander, H. Andersson, L. Andersson, R. Ansell, N. Kirsch, I. Nicholls and J. O’Mahony, J. Mol. Recogn., 2006, 19, 106-180.

[15] S. Chou and M. Syu, Biomaterials, 2009, 30, 1255-1262.

[16] N. Bereli, M. Andac, G. Baydemir, R. Say, I. Galaev and A. Denizli, J. Chromatogr, A, 2008, 1190, 18-26. 